\title{
RECOGNITION OF DEPRESSIVE SYMPTOMS BY PHYSICIANS
}

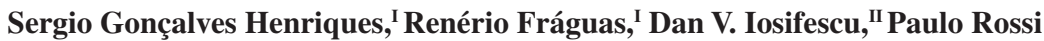 \\ Menezes, ${ }^{\text {III }}$ Mara Cristina Souza de Lucia, ${ }^{\mathrm{IV}}$ Wagner Farid Gattaz, ${ }^{\mathrm{I}}$ Milton \\ Arruda Martins $v$
}

doi: $10.1590 / \mathrm{S1807-59322009000700004}$

\begin{abstract}
Henriques SG, Fráguas R, Iosifescu DV, Menezes PR, Lucia MCS, Gattaz WF, Martins MA. Recognition of depressive symptoms by physicians. Clinics. 2009;64(7): 629-35.
\end{abstract}

OBJECTIVE: To investigate the recognition of depressive symptoms of major depressive disorder (MDD) by general practitioners. INTRODUCTION: MDD is underdiagnosed in medical settings, possibly because of difficulties in the recognition of specific depressive symptoms.

METHODS: A cross-sectional study of 316 outpatients at their first visit to a teaching general hospital. We evaluated the performance of 19 general practitioners using Primary Care Evaluation of Mental Disorders (PRIME-MD) to detect depressive symptoms and compared them to 11 psychiatrists using Structured Clinical Interview Axis I Disorders, Patient Version (SCID I/P). We measured likelihood ratios, sensitivity, specificity, and false positive and false negative frequencies.

RESULTS: The lowest positive likelihood ratios were for psychomotor agitation/retardation (1.6) and fatigue (1.7), mostly because of a high rate of false positive results. The highest positive likelihood ratio was found for thoughts of suicide (8.5). The lowest sensitivity, $61.8 \%$, was found for impaired concentration. The sensitivity for worthlessness or guilt in patients with medical illness was $67.2 \%$ (95\% CI, 57.4-76.9\%), which is significantly lower than that found in patients without medical illness, 91.3\% (95\% CI, 83.2-99.4\%).

DISCUSSION: Less adequately identified depressive symptoms were both psychological and somatic in nature. The presence of a medical illness may decrease the sensitivity of recognizing specific depressive symptoms.

CONCLUSIONS: Programs for training physicians in the use of diagnostic tools should consider their performance in recognizing specific depressive symptoms. Such procedures could allow for the development of specific training to aid in the detection of the most misrecognized depressive symptoms.

KEYWORDS: Internal medicine; Ambulatory care; Diagnosis; Depressive disorder; Primary care.

\section{INTRODUCTION}

Major depressive disorder (MDD) has been associated with increased morbidity ${ }^{1}$ and mortality, ${ }^{2}$ and is highly

\footnotetext{
I Department and Institute of Psychiatry, Hospital das Clinicas da Faculdade de Medicina da Universidade de São Paulo - São Paulo/SP, Brazil.

II Depression Clinical and Research Program, Massachusetts General Hospital, Harvard University - Boston, Massachusetts, USA.

III Department of Preventive Medicine, Faculdade de Medicina da Universidade de São Paulo - São Paulo/SP, Brazil.

${ }^{\text {IV }}$ Division of Psychology, Hospital das Clinicas da Faculdade de Medicina da Universidade de São Paulo - São Paulo/SP, Brazil.

${ }^{v}$ Department of Medical Clinic, Hospital das Clinicas da Faculdade de Medicina da Universidade de São Paulo - São Paulo/SP, Brazil.

Email: nupi1968@ hotmail.com

Received for publication on March 03, 2009

Accepted for publication on April 06, 2009
}

prevalent in primary care. ${ }^{3}$ Several initiatives to improve the recognition and treatment of depression by physicians have been successful ${ }^{4-9}$ however, there is still significant evidence for the underdiagnosis of depression in primary care. ${ }^{4,10}$ In addition to time constraints during the consultation and aspects related to physician training, ${ }^{7,11}$ several characteristics of depression may make its recognition difficult. For example, depression has been reported to be less likely to be recognized and diagnosed in patients who present somatic symptoms than in patients who present predominantly psychological symptoms. ${ }^{10,12}$ In contrast, the presence of medically unexplained somatic symptoms and hypochondriac worries have been reported to increase the rate of diagnosis. ${ }^{12}$ These studies suggest that recognition of certain depressive symptoms may be more difficult than 
others. We are not aware of any study that has specifically investigated this topic. Thus, this study was developed to investigate recognition of depressive symptoms of MDD by physicians using Primary Care Evaluation of Mental Disorders (PRIME-MD). ${ }^{13}$ The physicians' evaluations were compared to the evaluations of psychiatrists using Structured Clinical Interview Axis I, Patient Version (SCID-I/P). ${ }^{14}$

\section{METHODS}

\section{Subjects}

A total of 577 patients were evaluated for depression by their general practitioner, of whom 316 were also evaluated by a psychiatrist. This report includes all 316 subjects seen by both a general practitioner and a psychiatrist, in contrast to our previously published manuscript ${ }^{15}$ that included 240 subjects after excluding patients with missing data on medical status, health service utilization, and diagnostic criteria for MDD.

The 316 patients in this study were 18 years or older with a mean age of $40.8(+14.8)$ years. Most were female $(74 \%)$, married or living with a partner (51.3\%), and had five or less years of education (55.8\%). Except for higher rates of female gender and presence of medical illness, the group studied did not differ from the remaining 261 patients of the original sample of 577 subjects that were not included in this report (Table 1). We excluded patients that could not comprehend the questions and patients with health problems that limited the interview.

We examined the recognition of depressive symptoms by general practitioners in a cross sectional two-phase study at a teaching general outpatient unit (Ambulatório Geral Didático - AGD) of the Clinical Hospital of the Faculty of Medicine, Sao Paulo University (HC-FMUSP), São Paulo, Brazil. Details of the protocol have been published previously in a study focusing on the validity of diagnosing MDD and subsyndromal depression. 15 Briefly, 577 patients on their first visit to the outpatient unit were consecutively evaluated by the physician using Primary Care Evaluation of Mental Disorders (PRIME-MD). ${ }^{13}$ All patients with the diagnosis of MDD and a random sub-sample of those without MDD (odds of referral 1:4) were subsequently evaluated by a psychiatrist using SCID-I/P for DSM-IV disorders. ${ }^{14}$ The psychiatric evaluation was blinded and carried out immediately after the evaluation by the physicians. All patients signed a written informed consent form before being included in the study. The research protocol was approved by the Institutional Review Board of the Clinical Hospital of the Faculty of Medicine, Sao Paulo University HC-FMUSP.

A team of 11 psychiatrists and 19 physicians were recruited and trained. Two psychiatrists (RF and SGH) trained the physicians in PRIME-MD for DSM-III- $\mathrm{R}^{13}$ in a four-hour training program. The psychiatrists were trained by one of the authors (RF) in a six-hour program. All professionals received payment in currency for their work. All evaluations were carried out at the General Medicine Outpatient Unit.

Table 1 - Sociodemographic characteristics of the subjects, and comparison between patients interviewed by psychiatrists $(n=316$, included in this report $)$ and those not interviewed by psychiatrists $(n=261$, not included in this report $)$

\begin{tabular}{|c|c|c|c|c|c|}
\hline \multirow{3}{*}{ Characteristics } & \multicolumn{4}{|c|}{ Original sample $(n=577)$} & \multirow[b]{3}{*}{$\mathrm{p}$-value } \\
\hline & \multicolumn{2}{|c|}{ Studied group } & \multicolumn{2}{|c|}{ Group not included in this study } & \\
\hline & $\mathrm{n}=316$ & $\%$ & $\mathrm{n}=261$ & $\%$ & \\
\hline Gender & & & & & $0.020^{\mathrm{a}}$ \\
\hline Female & 234 & 74.0 & 170 & 65.1 & \\
\hline Marital Status & & & & & $0.476^{\mathrm{b}}$ \\
\hline Married/living with a partner & 162 & 51.3 & 132 & 50.6 & \\
\hline Never married & 68 & 21.5 & 70 & 27.8 & \\
\hline Divorced/separated/widowed & 57 & 18.0 & 42 & 16.1 & \\
\hline Not declared & 29 & 9.2 & 17 & 6.5 & \\
\hline Educational Level (years) & & & & & $0.174^{\mathrm{b}}$ \\
\hline 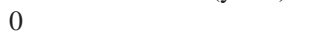 & 16 & 6.9 & 21 & 11.2 & \\
\hline $1-5$ & 114 & 48.9 & 98 & 52.4 & \\
\hline $6-10$ & 71 & 30.5 & 42 & 22.5 & \\
\hline More than 12 & 32 & 13.7 & 26 & 13.9 & \\
\hline Medical Disease & & & & & $0.003^{\mathrm{a}}$ \\
\hline Yes & 205 & 65.3 & 138 & 52.9 & \\
\hline No & 109 & 34.7 & 123 & 47.1 & \\
\hline Mean age (years) ( \pm S.D.) & 40.8 & 14.8 & 41.4 & 16.0 & $0.323^{\mathrm{c}}$ \\
\hline
\end{tabular}


PRIME-MD for DSM-III-R, although comprised of structured questions, permits further investigation and inquiries according to the judgment of the interviewer. It is divided into five modules (mood, anxiety, somatoform, alcohol and eating disorders) covering the most common disorders found in primary care. Nine questions investigate the main depressive symptoms and the presence of MDD according to the DSM-III-R criteria. All diagnoses were determined from the questionnaire by answers of 'yes' or 'no'. ${ }^{13}$ PRIME-MD was translated into Portuguese by two of the authors (RF and SGHJ). The Portuguese version was back-translated into English and submitted for the approval of the author (Dr. Spitzer), whose suggestions were incorporated into the final Portuguese version. For the present study, we utilized the answers to the PRIME-MD mood module (which covers the diagnosis of MDD). The answers to the psychiatrist-administered SCID I/P ${ }^{14}$ were used as a comparison. The mood modules of PRIME-MD and SCID I/P cover the same DSM-III-R items for the diagnosis of MDD. The physicians evaluated the presence of a medical illness by an open interview and a check list with 13 possible categories of medical illness including: cardiovascular, infectious, endocrine, neurological, rheumatic, gastrointestinal, dermatologic, musculoskeletal, gynecologic, oncological, allergic, and immunologic illnesses. For this study, we included all subjects having any medical condition in any of the 13 categories in the group with medical illness(es).

The statistical analysis was performed to investigate three aspects: 1- The ability of the physicians to recognize each depressive symptom; 2- The influence of a medical illness on the recognition of each depressive symptom; and 3- The influence of gender on the recognition of each depressive symptom.

Following the SCID I/P guidelines, we counted symptoms coded "3" (threshold) as present and symptoms coded " 1 " or " 2 " as absent.

To investigate the performance of the physicians at recognizing each depressive symptom, we calculated sensitivity, specificity, false positives and negatives, true positives and negatives, and positive and negative likelihood ratios for each depressive symptom evaluated by the physicians using PRIME-MD and compared them to the evaluations of the psychiatrists using SCID I/P (standard criteria). The positive likelihood ratio is defined as the likelihood of a positive evaluation of a depressive symptom in a patient with the symptom divided by the likelihood of a positive evaluation in a patient without that depressive symptom. For example, let us consider the symptom of insomnia and the hypothetical situation in which the physicians correctly detect $90 \%$ of the patients with true insomnia (sensitivity), and incorrectly detect $15 \%$ of the subjects without insomnia as having insomnia (false positive $=1$-specificity). The physicians were six times as likely to recognize a patient with insomnia as opposed to a patient without insomnia (i.e., 90 divided by 15), which is the likelihood ratio for a positive test result. We chose the likelihood ratio as it is not influenced by symptom prevalence, thus allowing for a closer evaluation of physician performance.

To investigate the influence of patient gender and the presence of medical illness on the recognition of depressive symptoms, we measured specificity and sensitivity for each depressive symptom separately in four groups defined by gender and the presence or absence of medical disease.

Analyses were performed using Stata version 8.2 for Windows. The accepted two-sided significance level was alpha 0.05 . Given the preliminary nature of this study, we did not use adjustments for multiple comparisons.

\section{RESULTS}

In this study, which compared the performance of physicians using PRIME-MD to identify depressive symptoms in 316 outpatients to that of psychiatrists using SCID, we found the lowest sensitivities for the symptoms of impaired concentration $(61.8 \%)$, appetite or weight change (72.2\%), and insomnia or hypersomnia (74.1\%) (Table $2)$. False negative results were particularly common for impaired concentration $(n=68)$, depressed $\operatorname{mood}(n=53)$, and appetite or weight changes $(n=52)$ (Table 2).

The depressive symptoms with the lowest positive likelihood ratios were psychomotor agitation/retardation (1.6) and fatigue (1.7) (Table 2).

The symptom with the lowest sensitivity for females and males both with and without medical conditions was impaired concentration (Tables 3 and 4). Thoughts of death/ suicide was the symptom with the greatest difference in the sensitivity between males and females, $68.1 \%$ vs. $77.0 \%$ (Table 3), respectively. Even in this case, the confidence intervals for the two genders overlapped, indicating that the difference was not statistically significant.

Although most of the confidence intervals overlapped, the sensitivity for almost all of the depressive symptoms was numerically lower in the group with medical disease than in the group without medical disease. The exception was "thoughts of death," which were more readily detected by physicians in subjects with medical illnesses. The sensitivity for worthlessness or guilt exhibited the greatest difference between patients with or without medical disease, $67.2 \%$ vs. $91.3 \%$, respectively. The difference was statistically significant (Table 4). 
Table 2 - Primary care physicians' recognition of depressive symptoms: sensitivity, specificity, true positive, true negative, false positive, false negative, positive and negative likelihood ratios $(n=316)$

\begin{tabular}{|c|c|c|c|c|c|c|c|c|c|c|}
\hline Depressive symptoms & sensitivity & $95 \% \mathrm{CI}$ & specificity & $95 \% \mathrm{CI}$ & $\begin{array}{c}\text { True } \\
\text { positive }\end{array}$ & $\begin{array}{c}\text { True } \\
\text { negative }\end{array}$ & $\begin{array}{c}\text { False } \\
\text { positive }\end{array}$ & $\begin{array}{c}\text { False } \\
\text { negative }\end{array}$ & $\begin{array}{l}\text { Positive } \\
\text { likelihood } \\
\text { ratio }\end{array}$ & $\begin{array}{c}\text { Negative } \\
\text { likelihood } \\
\text { ratio }\end{array}$ \\
\hline Depressed mood & 72.9 & $(67.3-78.5)$ & 70.4 & $(57.2-83.6)$ & 176 & 27 & 14 & 53 & 2.5 & 0.4 \\
\hline Loss of interest/pleasure & 77.3 & $(71.7-82.9)$ & 61.1 & $(50.0-72.1)$ & 166 & 41 & 29 & 44 & 2.0 & 0.4 \\
\hline Appetite/weight change & 72.2 & $(66.0-78.3)$ & 85.4 & $(78.1-92.7)$ & 140 & 73 & 13 & 52 & 4.9 & 0.3 \\
\hline Insomnia/hypersomnia & 74.1 & $(67.9-80.3)$ & 75.0 & $(66.6-83.3)$ & 134 & 75 & 25 & 47 & 3.0 & 0.3 \\
\hline $\begin{array}{l}\text { Psychomotor agitation/ } \\
\text { retardation }\end{array}$ & 80.0 & $(73.0-87.0)$ & 48.5 & $(40.4-56.6)$ & 98 & 60 & 68 & 23 & 1.6 & 0.4 \\
\hline Fatigue & 84.5 & $(79.9-89.1)$ & 50.0 & $(37.3-62.6)$ & 199 & 28 & 30 & 34 & 1.7 & 0.3 \\
\hline Worthlessness/guilt & 75.4 & $(68.4-82.5)$ & 61.4 & $(53.5-69.3)$ & 101 & 83 & 52 & 33 & 2.0 & 0.4 \\
\hline Impaired concentration & 61.8 & $(55.0-68.6)$ & 76.2 & $(67.7-84.7)$ & 109 & 71 & 22 & 68 & 2.6 & 0.5 \\
\hline Thoughts of death/suicide & 77.0 & $(68.3-85.6)$ & 90.9 & $(86.6-95.2)$ & 63 & 159 & 15 & 20 & 8.5 & 0.3 \\
\hline
\end{tabular}

Table 3 - Primary care physicians' recognition of depressive symptoms: sensitivity and specificity according to gender ( $\mathrm{n}=316$ )

\begin{tabular}{|c|c|c|c|c|c|c|c|c|}
\hline \multirow[b]{2}{*}{ Depressive symptoms } & \multicolumn{4}{|l|}{ Female } & \multicolumn{4}{|l|}{ Male } \\
\hline & Sensitivity & $95 \% \mathrm{CI}$ & Specificity & $95 \% \mathrm{CI}$ & Sensitivity & $95 \% \mathrm{CI}$ & Specificity & $95 \% \mathrm{CI}$ \\
\hline Depressed mood & 75.7 & $(69.3-82.0)$ & 61.2 & $(48.1-74.2)$ & 78.8 & $(67.7-89.9)$ & 66.7 & $(49.8-83.5)$ \\
\hline Loss of interest/pleasure & 77.5 & $(71.3-83.8)$ & 52.0 & $(39.6-64.4)$ & 84.6 & $(73.3-95.9)$ & 60.5 & $(45.9-75.1)$ \\
\hline Appetite/weight change & 71.1 & $(63.7-78.6)$ & 73.1 & $(63.9-82.3)$ & 76.6 & $(64.6-88.6)$ & 79.2 & $(65.5-92.9)$ \\
\hline Insomnia/hypersomnia & 77.3 & $(70.4-84.3)$ & 65.5 & $(56.0-75.0)$ & 65.9 & $(51.3-80.4)$ & 65.9 & $(51.3-80.4)$ \\
\hline Psychomotor agitation/retardation & 81.3 & $(73.6-89.1)$ & 40.9 & $(32.6-49.2)$ & 80.0 & $(64.3-95.7)$ & 54.4 & $(41.5-67.3)$ \\
\hline Fatigue & 85.7 & $(80.6-90.8)$ & 42.3 & $(28.9-55.7)$ & 84.3 & $(74.3-94.3)$ & 48.4 & $(30.8-66.0)$ \\
\hline Worthlessness/guilt & 74.8 & $(66.7-83.0)$ & 55.0 & $(46.2-63.7)$ & 76.4 & $(59.9-92.9)$ & 60.1 & $(47.3-72.9)$ \\
\hline Impaired concentration & 61.6 & $(53.6-69.5)$ & 67.4 & $(57.7-77.2)$ & 59.5 & $(43.2-75.7)$ & 67.9 & $(54.5-81.3)$ \\
\hline Thoughts of death/suicide & 77.0 & $(66.9-87.1)$ & 83.2 & $(77.4-88.9)$ & 68.1 & $(45.1-91.0)$ & 87.8 & $(80.0-95.7)$ \\
\hline
\end{tabular}

$\mathrm{CI}=$ confidence interval

\section{DISCUSSION}

Using 316 subjects from an outpatient unit of a teaching general hospital, we investigated the performance of general practitioners using PRIME-MD to recognize depressive symptoms of MDD and compared their performance to that of psychiatrists using SCID I/P. Of note, the highest likelihood ratio was 8.5 for thoughts of suicide. Although it did not achieve the level considered to be strong clinical evidence of the presence of a symptom (above
10, as proposed by Deeks, 2004), ${ }^{16}$ this result encourages physicians to investigate suicidal ideation. On the other hand, the lowest positive likelihood ratios were for fatigue and psychomotor agitation/retardation. The sensitivities for these symptoms were relatively high. In addition, a high rate of false positives (i.e. physicians frequently considered these symptoms present when they, in fact, were absent) but not false negatives was found. This relatively high sensitivity for fatigue and psychomotor agitation/retardation contrasts with previous studies in which physicians had lower rates 
Table 4 - Primary care physicians recognition of depressive symptoms: sensitivity and, specificity according to presence or not of medical illness $(\mathrm{n}=316)$

\begin{tabular}{|c|c|c|c|c|c|c|c|c|}
\hline \multirow[b]{2}{*}{ Depressive symptoms } & \multicolumn{4}{|c|}{ Absence of medical illness } & \multicolumn{4}{|c|}{ Presence of medical illness } \\
\hline & Sensitivity & $95 \% \mathrm{CI}$ & Specificity & $95 \% \mathrm{CI}$ & Sensitivity & $95 \% \mathrm{CI}$ & Specificity & $95 \% \mathrm{CI}$ \\
\hline Depressed mood & 79.2 & $(70.4-88.0)$ & 54.9 & $(36.7-73.0)$ & 75.2 & $(68.3-82.2)$ & 67.6 & $(55.2-79.9)$ \\
\hline Loss of interest/pleasure & 84.8 & $(76.3-93.4)$ & 43.7 & $(28.8-58.7)$ & 76.4 & $(69.5-83.3)$ & 63.5 & $(51.6-75.4)$ \\
\hline Appetite/weight change & 79.9 & $(70.2-89.6)$ & 61.9 & $(47.7-76.2)$ & 68.1 & $(60.0-76.2)$ & 81.1 & $(72.4-89.7)$ \\
\hline Insomnia/hypersomnia & 78.5 & $(68.5-88.5)$ & 65.2 & $(51.5-79.0)$ & 72.2 & $(64.4-80.4)$ & 65.8 & $(56.0-75.6)$ \\
\hline Psychomotor agitation/retardation & 84.0 & $(72.3-95.7)$ & 38.4 & $(27.2-49.7)$ & 79.8 & $(71.2-88.4)$ & 48.0 & $(39.1-56.8)$ \\
\hline Fatigue & 85.5 & $(77.6-93.4)$ & 31.4 & $(16.0-46.8)$ & 84.8 & $(79.2-90.4)$ & 53.1 & $(39.1-67.0)$ \\
\hline Worthlessness/guilt & 91.3 & $(83.2-99.4)$ & 53.8 & $(41.7-66.0)$ & 67.2 & $(57.4-76.9)$ & 57.7 & $(48.7-66.7)$ \\
\hline Impaired concentration & 65.8 & $(54.0-77.5)$ & 61.8 & $(47.9-75.7)$ & 58.9 & $(50.0-67.9)$ & 69.8 & $(60.3-79.3)$ \\
\hline Thoughts of death/suicide & 69.7 & $(54.2-85.3)$ & 81.7 & $(73.1-90.4)$ & 79.2 & $(67.8-90.6)$ & 85.4 & (79.9-91.0) \\
\hline
\end{tabular}

$\mathrm{CI}=$ confidence interval

of recognition of depression in patients with somatic presentation of depressive symptomatology. ${ }^{10,17}$ The design of our study, in which physicians were active participants who were specifically trained to diagnose depression and to utilize PRIME-MD (which includes fatigue and psychomotor agitation/retardation as depressive symptoms) may have contributed to this high level of sensitivity. In our study, physicians were more likely to recognize fatigue and psychomotor agitation/retardation as depressive symptoms because of exceptional concern over missing a diagnosis of MDD. This worry could have overcome the normalizing symptom attribution described in studies of primary care patients. ${ }^{10,18-25}$ In previous studies, low sensitivity and specificity rates for recognition of somatic depressive symptoms have been attributed, at least in part, to the use of instruments that exclude somatic symptoms. ${ }^{10}$ For example, the Hampshire Depression Project used the Hospital Anxiety and Depression Scale (HADS), which excludes somatic symptoms, to evaluate depression; it did not achieve a significant improvement in the recognition of depressive symptoms. ${ }^{20}$ However, the high rate of false positive results for both fatigue and psychomotor agitation/ retardation observed in our study (which used PRIME-MD, an instrument that captures somatic symptoms) indicates that the simple inclusion of somatic symptoms in the list of symptoms to diagnose depression may not completely solve the problem. The tendency of patients with low educational levels to over-report complaints in the absence of symptoms ${ }^{26}$ may lead physicians to make false positive diagnoses, and thus might partially explain this finding.
The lowest sensitivity observed in our study was for the symptom of impaired concentration. This trend held true in the total sample and in the groups defined by gender and by the presence or absence of a medical condition. Although impaired concentration may also be present in other psychiatric disorders, it has been reported to be significantly and independently associated with the diagnosis of MDD. ${ }^{21}$ In addition, neurocognitive deficit has been considered an objective marker of the cerebral dysfunction of depression, ${ }^{22,23}$ and it has been associated with a history of psychiatric hospitalizations..$^{24,27}$ Although the modules of SCID I/P and PRIME-MD for the diagnosis of MDD use the same criteria, they differ with respect to impaired concentration. Whereas PRIME-MD includes only impaired concentration to fulfill the criterion, SCID I/P includes the presence of indecision as an alternative to impaired concentration for fulfilling the criterion. It is possible to speculate that physicians consider impaired concentration as a relatively normal stress-related complaint and do not recognize it as a possible depressive symptom. However, because of the above-mentioned difference between PRIMEMD and SCID-IP, we cannot exclude that this low sensitivity for impaired concentration results from the non-inclusion of indecision as an alternative for impaired concentration in PRIME-MD. Indecision has been reported to be a good marker of depression severity, independent of the presence of a medical illness. ${ }^{1,25,28}$ In our sample, 177 (65\%) patients had impaired concentration or indecision according to the psychiatrists using SCID-IP, and the physicians missed 68 (38\%) of these cases. Our sense is that this item in PRIME- 
MD deserves revision, and a new version should include the symptoms impaired concentration and indecision.

Physicians have a lower sensitivity for detecting worthlessness or guilt in patients with medical disease than in subjects without medical disease $(67.2 \%$ versus $91.3 \%)$. Since the presence of a medical illness is probably not associated with differences in the frequency of symptoms used to diagnose major depressive disorder ${ }^{27}$ our finding may reflect the tendency of physicians to consider psychological depressive symptoms (i.e., worthlessness or guilt) as normal psychological reactions to medical disease. Previous studies have reported a lower recognition of depression by general practitioners in patients with medical illness, ${ }^{28,29}$ and in patients with more severe medical illness. ${ }^{1,29}$

Our study has some limitations that should be considered. First, although the 19 participant physicians received payment for their evaluations, we can not exclude the possibility that this group is overpopulated with physicians more interested in psychiatry and possibly more prone to adequately evaluate the depressive symptoms. The physicians were all from the HC-FMUSP, and the peculiarities of medical education in the undergraduate and resident programs restrict the generalization of our findings to physicians from other centers. The symptomatologic depressive profile of our subjects may have specificities that limit the generalization of our results to patients from other centers and units. However, the strategy of investigating the recognition of each specific symptom that we used in our study could be a promising mechanism by which to improve physicians' skills for the diagnosis of depression.

In conclusion, this study of physicians using PRIME-MD to detect depressive symptoms compared to psychiatrists using SCID found low positive likelihood ratios for fatigue and psychomotor agitation/retardation, and low sensitivity for impaired concentration. These findings suggest that the physicians' ability to recognize depressive symptoms may vary across the depressive symptomatology independent of their psychological or somatic nature. Thus, programs aiming at improving the detection of depression in medical settings should consider physicians' clinical judgment, as has been previously proposed ${ }^{30}$ and focus on their performance at recognizing specific depressive symptoms. Our sense is that repeating the training of the participant physicians and discussing the results and the underlying causes of the discrepancies could optimize efforts and considerably enhance the physicians' skills.

\section{ACKNOWLEDGMENTS}

The study received financial support from The State of São Paulo Research Foundation (FAPESP, project 00669-9), São Paulo, Brazil.

\section{REFERENCES}

1. Coulehan JL, Schulberg HC, Block MR, Janosky JE, Arena VC. Medical comorbidity of major depressive disorder in a primary medical practice. Arch Intern Med. 1990;150:2363-7.

2. Davidson JR, Meltzer-Brody SE. The underrecognition and undertreatment of depression: what is the breadth and depth of the problem? J Clin Psychiatry. 1999;60 Suppl 7:4-9; discussion 10-1.

3. Ansseau M, Dierick M, Buntinkx F, Cnockaert P, De Smedt J, Van Den Haute $\mathrm{M}$, et al. High prevalence of mental disorders in primary care. J Affect Disord. 2004;78:49-55.

4. Kessler D, Bennewith O, Lewis G, Sharp D. Detection of depression and anxiety in primary care: follow up study. BMJ. 2002;325:1016-7.

5. Pignone MP, Gaynes BN, Rushton JL, Burchell CM, Orleans CT, Mulrow CD, et al. Screening for depression in adults: a summary of the evidence for the U.S. Preventive Services Task Force. Ann Intern Med. 2002;136:765-76.

6. Simon GE, Fleck M, Lucas R, Bushnell DM. Prevalence and predictors of depression treatment in an international primary care study. Am J Psychiatry. 2004;161:1626-34.

7. van Os TW, Ormel J, van den Brink RH, Jenner JA, Van der Meer K, Tiemens BG, et al. Training primary care physicians improves the management of depression. Gen Hosp Psychiatry. 1999;21:168-76.
8. Rix S, Paykel ES, Lelliott P, Tylee A, Freeling P, Gask L, et al. Impact of a national campaign on GP education: an evaluation of the Defeat Depression Campaign. Br J Gen Pract. 1999;49:99-102.

9. Stevens L, Kinmonth AL, Peveler R, Thompson C. The Hampshire Depression Project: development and piloting of clinical practice guidelines and education about depression in primary health care. Med Educ. 1997;31:375-9.

10. Tylee A, Gandhi P. The importance of somatic symptoms in depression in primary care. Prim Care Companion J Clin Psychiatry. 2005;7:167-76.

11. Tylee A. Depression in the community: physician and patient perspective. J Clin Psychiatry. 1999;60 Suppl 7:12-6; discussion 17-8.

12. Kirmayer LJ, Robbins JM, Dworkind M, Yaffe MJ. Somatization and the recognition of depression and anxiety in primary care. Am J Psychiatry. 1993;150:734-41.

13. Spitzer RL, Williams JB, Kroenke K, Linzer M, deGruy FV, Hahn SR, et al. Utility of a new procedure for diagnosing mental disorders in primary care. The PRIME-MD 1000 study. JAMA. 1994;272:1749-56.

14. First MBSR, Gibbson M, Williams JBW. Structured clinical interview for axis I DSM-IV disorders (Version 2.0)-patient edition. New York:Biometrics Research Department, New York State Psychiatric Institute. 1995. 
15. Fraguas R, Henriques SG, De Lucia MS, Iosifescu DV, Schwartz FH, Menezes PR, et al. The detection of depression in medical setting: a study with PRIME-MD. J Affect Disord. 2006;91:11-7.

16. Deeks JJ, Altman DG. Diagnostic tests 4: likelihood ratios. BMJ. 2004;329:168-9.

17. Parker G, Parker K. Influence of symptom attribution on reporting depression and recourse to treatment. Aust N Z J Psychiatry. 2003;37:469-74.

18. Bower P, West R, Tylee A, Hann M. Symptom attribution and the recognition of psychiatric morbidity. J Psychosom Res. 2000;48:157-60.

19. Kessler D, Lloyd K, Lewis G, Gray DP. Cross sectional study of symptom attribution and recognition of depression and anxiety in primary care. BMJ. 1999;318:436-9.

20. Thompson C, Kinmonth AL, Stevens L, Peveler RC, Stevens A, Ostler $\mathrm{KJ}$, et al. Effects of a clinical-practice guideline and practice-based education on detection and outcome of depression in primary care: Hampshire Depression Project randomised controlled trial. Lancet. 2000;355:185-91.

21. Zimmerman M, McGlinchey JB, Young D, Chelminski I. Diagnosing major depressive disorder IX: are patients who deny low mood a distinct subgroup? J Nerv Ment Dis. 2006;194:864-9.

22. Porter RJ, Gallagher P, Thompson JM, Young AH. Neurocognitive impairment in drug-free patients with major depressive disorder. Br J Psychiatry. 2003;182:214-20.

23. Pardo JV, Pardo PJ, Humes SW, M IP. Neurocognitive dysfunction in antidepressant-free, non-elderly patients with unipolar depression: alerting and covert orienting of visuospatial attention. J Affect Disord. 2006;92:71-8.
24. Purcell R, Maruff P, Kyrios M, Pantelis C. Neuropsychological function in young patients with unipolar major depression. Psychol Med. 1997;27:1277-85

25. Clark DC, vonAmmon Cavanaugh S, Gibbons RD. The core symptoms of depression in medical and psychiatric patients. J Nerv Ment Dis. $1983 ; 171: 705-13$

26. Ludermir $\mathrm{AB}$, Lewis $\mathrm{G}$. Investigating the effect of demographic and socioeconomic variables on misclassification by the SRQ-20 compared with a psychiatric interview. Soc Psychiatry Psychiatr Epidemiol. 2005;40:36-41.

27. Yates WR, Mitchell J, Rush AJ, Trivedi MH, Wisniewski SR, Warden $\mathrm{D}$, et al. Clinical features of depressed outpatients with and without cooccurring general medical conditions in STAR*D. Gen Hosp Psychiatry. 2004;26:421-9.

28. Freeling P, Rao BM, Paykel ES, Sireling LI, Burton RH. Unrecognised depression in general practice. Br Med J (Clin Res Ed). 1985;290:18803.

29. Tylee AT, Freeling P, Kerry S. Why do general practitioners recognize major depression in one woman patient yet miss it in another? $\mathrm{Br} \mathrm{J}$ Gen Pract. 1993;43:327-30

30. Kendrick T, Dowrick C, McBride A, Howe A, Clarke P, Maisey S, et al. Management of depression in UK general practice in relation to scores on depression severity questionnaires: analysis of medical record data. Bmj. 2009;338:b750. 
